\title{
Windows software for enhanced studying and testing knowledge in optics
}

Sergey Stafeev, Vladimir Michnovetz, Alexander Khmaladze, Alexander Zinchik

Sergey C. Stafeev, Vladimir J. Michnovetz, Alexander T. Khmaladze, Alexander A. Zinchik, "Windows software for enhanced studying and testing knowledge in optics," Proc. SPIE 2525, 1995 International Conference on Education in Optics, (13 October 1995); doi: 10.1117/12.224052

Event: SPIE's 1995 International Symposium on Optical Science, Engineering, and Instrumentation, 1995, San Diego, CA, United States 
Windows software for enhanced studying and testing knowledge in optics

Serguej C. Stafeev, Vladimir J. Michnovetz, Alexander T. Khmaladze, Alexander A. Zinchik

General Physics Department of St.Petersburg Institute of Fine Mechanics \& Optics (PIFMO)

14 Sablinskaya st., St. Petersburg, 197101 Russia

ph: (812)238-8727, fax:(812)232-7622, e-mail: physics@ipmo.spb.su

\begin{abstract}
We describe recent advances in the development of the original software for studying optics on the General Physics level. Two types of Windows ${ }^{\mathrm{TM}}$ software are reported: the guide-programs for simulation the basic optical experiments and the multichoice test-programs for teachers (to create tests) and for students (to check their knowledge). Application of guide-programs combined with image-files from CCD-camera is presented in two modes: with real equipment and simulation with empirical data-files. The testing system uses GRE approach and accompanied a lot of pictures with main optical circuits or charts. Some illustrations with real screen views for basic optical phenomena are presented.
\end{abstract}

\title{
INTRODUCTION
}

This contribution is closely connected with our report "Concept of Modern Optical Training Labs for Optical Engineering Students" in the same volume [1]. The block module principle of laboratory equipment construction is used for software also.working out. Exercises scenario includes the following: introduction, preliminary (or final) student's test, laboratory exercise fulfillment by guide-program, working with a simulating programs. A test program is performed by multichoice principle or by freeconstructing answer to questions of different sorts. A main program of laboratory exercise doing or the so-called guide-program contains some directions to make up necessary scheme to be measured, gives in interactive regime a discussion of the experimental parameters, takes on student's results of measuring, makes control an interface and carries out statistical treating of dates. This program does also some tables, provides the better graphic approximation, fulfills total results for the account. The flexible construction of guide-program permits to instructor to vary both the total volume of experiments and regimes of their executing. That depends on the duration of study, on the level of student's preparation to the work doing and on student's future specialty. The third part of original software is the Digital Image Files Collection, which includes more than two hundreds images from real test-objects (diffraction, interference, polarization phenomena etc. [1]) and pictures with main optical circuits.

\section{TESTING PROGRAMS}

Test-files are created by TestMaker program (Fig.1) and used for testing students without limit of time on 5 randomly chosen questions in a certain topic. The results of the answers are indicated by sound signal and graphic icon also. Questions in test-files have no any coefficients of difficulty and, therefore students get the marks simply according to the number of right answers. Text of the questions and answers are typed in the appropriate windows by keyboard in Russian and English mode by Sans Serif Physics font, which is added to this software. The font is based on standard Sans Serif font and that is why, one can type the text in any Windows text editors (Cardfile, Notepad, Write, etc.), and use clipboard to transmit them into the test-files. Sans Serif Physics font include Sans Serif font letters and, additionally, some special symbols, needed for test-files (e.g., Greece letters, math symbols, etc.). One can use hot key combinations to add them in text. These combinations can be viewed by CharacterMap. It is noted the size of the text windows is limited, but the question windows have a scrollbar.

In TestMaker pictures, images and frames are also available. They can be painted in any Windows graphic editors (e.g., Paintbrush) or received by video camera in BMP-format. Optimum picture size is $252 * 162$ pixels. The pictures, images and frames can be color, black-white or gray. The color palette depends on computer, hence it is recommended to use the basic colors. The relation between picture and a question of the test-file is made automatically by using Picture button. Then, one can choose the appropriate BMP-file, which now had to be in the same directory. After that, test-files will include the name to BMP-file. When the text and graphic windows are done, one has to indicate the right answer, and then goes to the next question. The whole file is done when all questions are ready and saved as.TST file. Edition of existing file is made the same way. Obviously, if is possible to edit either text and picture or both. For example, one can repaint the pictures, or simply add other BMP files to the test-files. 

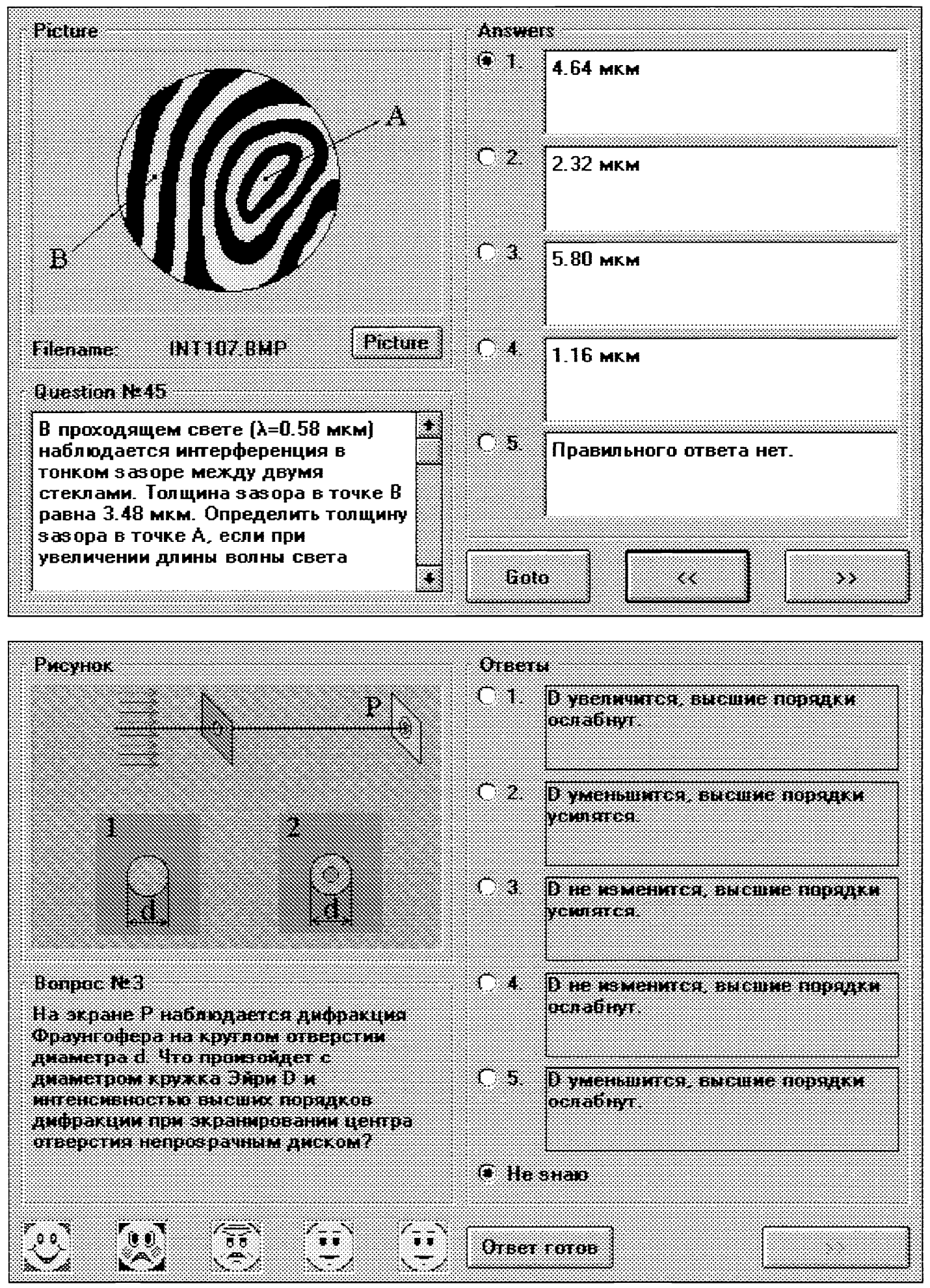

Fig. 1 Examples of TestMaker File (above, Equal Thickness Interference) and Testing Sheet (below, Fraunhofer Diffraction). Five icons in the bottom show one true answer, one wrong answer and thinking about third question. 


\section{CHECKING PROGRAMS}

Check-files are created by the Check-Maker program (Fig.2) and used for testing students with limit of time on a certain number of randomly chosen questions. The results of the answers are indicated by sound signal and graphic icon and also change a total score. Questions in check-files have coefficients of difficulty, which depend on a percentage of right answers, given on expert testing and also difficulty of the question. Right answer on the question means the score increases on the value of this coefficient, wrong answer means the score decreases on one fourth of the value. The same method is used in American GRE (Graduate Record Examination) tests.

The procedure of producing pictures and text windows of check-files is completely the same as test-files. Check-files are saved as. CHK files. Certainly, it is possible to transmit text information between test- and check-files. The number of questions in check-files is simply the number of filled fields, varying from 1 to 100. This number is indicated in Questions field. The weight of the question is indicated in Weight edit window and varying from 1 to 999. Practically, weight is usually varying from 2 to 20 (and is even number, because if answer is wrong the score will be multiple by one half). Sure, it is possible to edit the weight coefficients. In this case the number in Total weight field changes automatically. One can set the total testing time for the certain check-file in the Setting menu. This time can be varying from 1 to 999 minutes, but practically is 10-15 minutes for current control and 180-200 minutes for final graduate tests.

After finishing of text-files and their BMP-files, the test- and check-files are put into the separated directory, where TestShell and CheckShell exist. These programs support the testing procedure and collect results. Each of the programs starts by double-click on its icon in Windows group (installation is automatic). It is possible to use different icons for every physical topic, which call the same EXE-files with different subdirectories.

After the TestShell program is started from Program Manager's group (see Fig.2 in [1]) or by File Manager, a supervisor opens a test-file and then a student can "push" the button on the left down corner of the screen, and goes to the first question. After finding an answer, the student indicates it by click consequence button on a screen by mouse. It is possible to refuse to answer and simply go to next question. At the moment, all the Windows Accessories (e.g., calculator) are available. When the test is passed, the click on left down button opens window, which asks student to call the supervisor. After the CheckShell program is started and check-file is opened, a student can see an information about the number of questions, testing time and maximum total score. Then, one goes to first the question, preliminary registries his/her group number and last name. Testing procedure seems the same like TestShell. The results are counted by adding the weight of the answer to the total score if the answer is right and subtracting the one fourth weight if the answer is wrong. If the students refuse to answer, the total score remains the same. It is possible to provide testing on several computers at the same time, because the order of question is random. Test is finished, when all questions are passed or the time is over.

Collection of results is used for two main purposes. First, one can collect statistical data about different test of a certain student, which helps to decide what topics are tough for him/her and what topics are had to be studied individually. Second is the collection the data about one testing of a number of students on a certain topic. In this case, teacher may decide, what topics are easy to understand, what are not, and also make some remarks about weight of the question. For registration one should prepare group lists, types them in Notepad and puts in subdirectory with CheckShell program. The group lists include student's names in row in alphabet order. They save as files, which named as group. The lists can be made longer and shorter anytime.

Current control testing was made approximately one time per 2-3 weeks and was connected with current lecture material. There were 5-6 check-files on each test, with 12-16 questions in each file. Total scores (maximum) were ranged from 60 to 80 and times limit $30 \ldots 40 \mathrm{~min}$. Check-files were chosen according to levels of testing groups and contains more questions for easy and less for tough questions to keep maximum score approximately the same. The results were transmitted to Excel 4.0 and processed statistically to obtain average score and standard deviation for each group. This data changed automatically when new data obtained. Final control testing may be prepared by transmittance the 

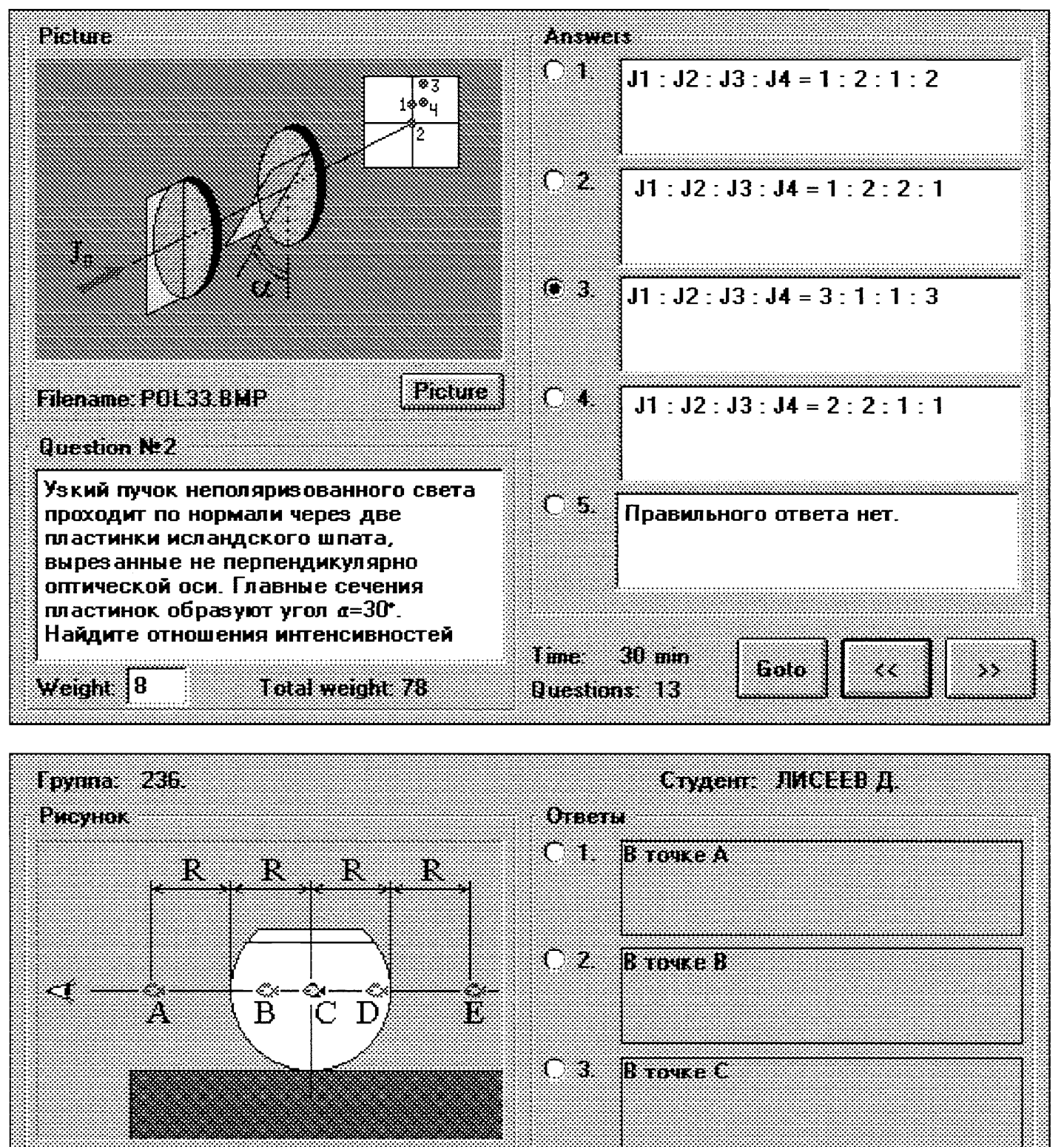

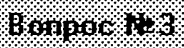

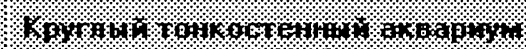

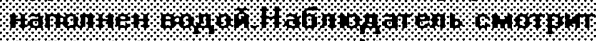

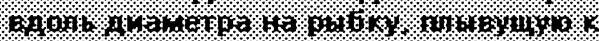

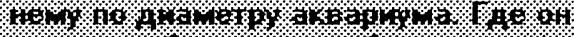

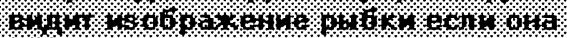

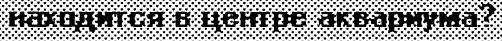

\subsection{0}

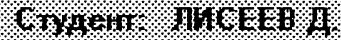

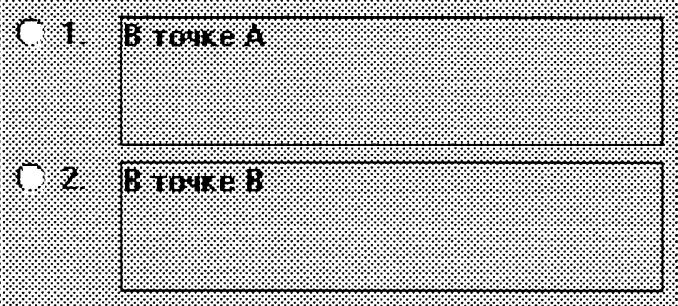

$1.1 .1 \% 18010 \%$

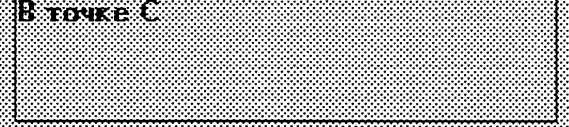

$10 \% 1010 \%$

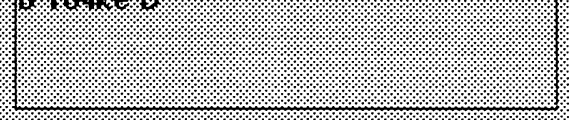

$10 \% 1010 \%$

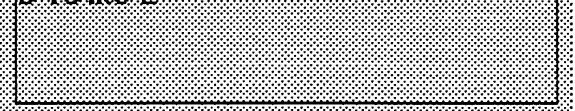

010001010

16710701001-1.

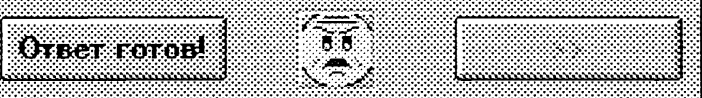

Fig.2 Examples of CheckMaker File (above, Polarization Phenomena) and Checking Sheet (below, Ray Optics). Question number, Timer data, total and current Score are shown. 

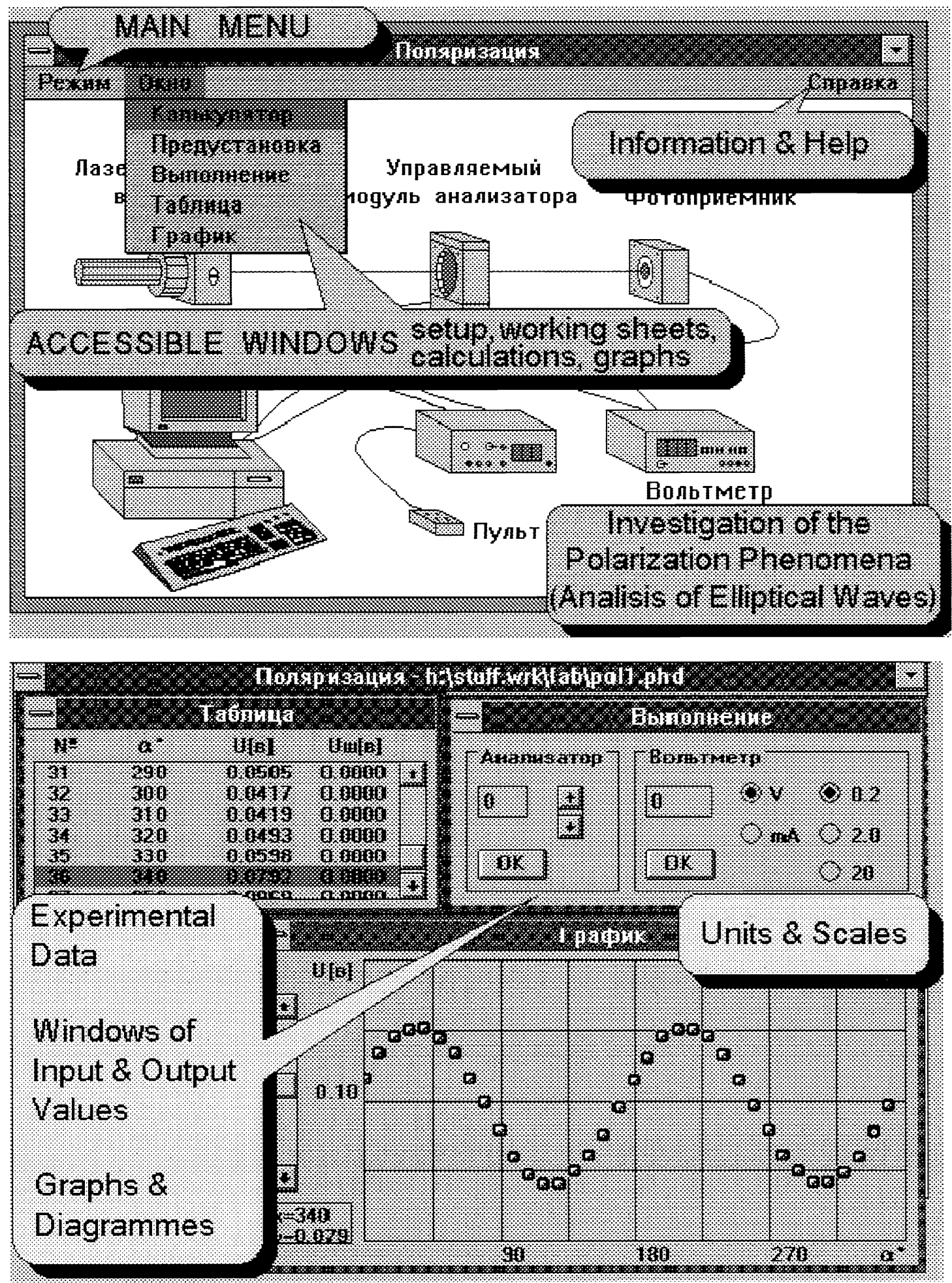

Fig. 3 View of the Screen (above) and Working Sheet (below) of LabShell programm for studying Polarization Phenomena by using Laser Beam and different phase Objects 


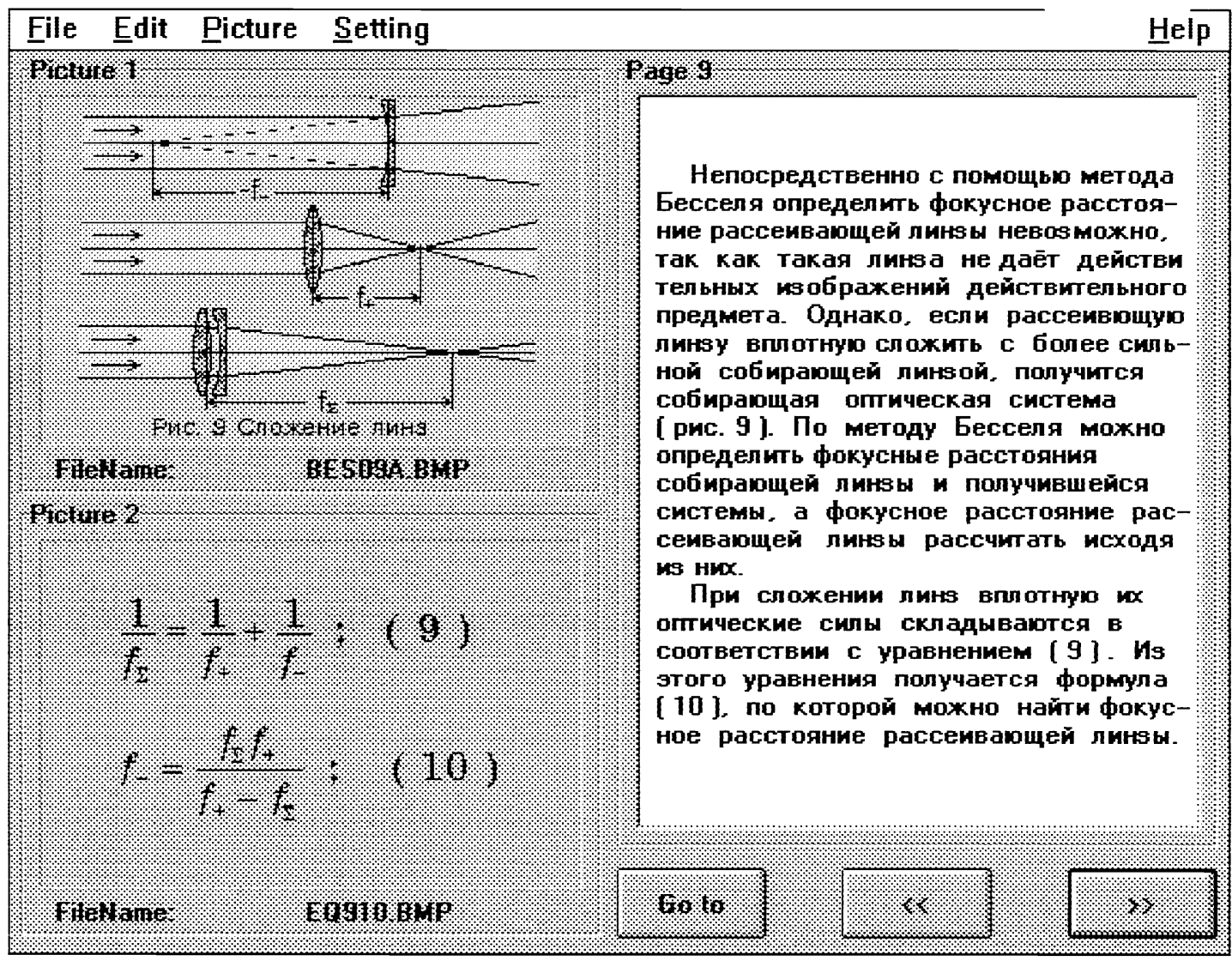

Fig. 4.One page of GuideShell-program: Bessel method for Focal Leight Determination ( Single Lenses and Lenses Sistems )

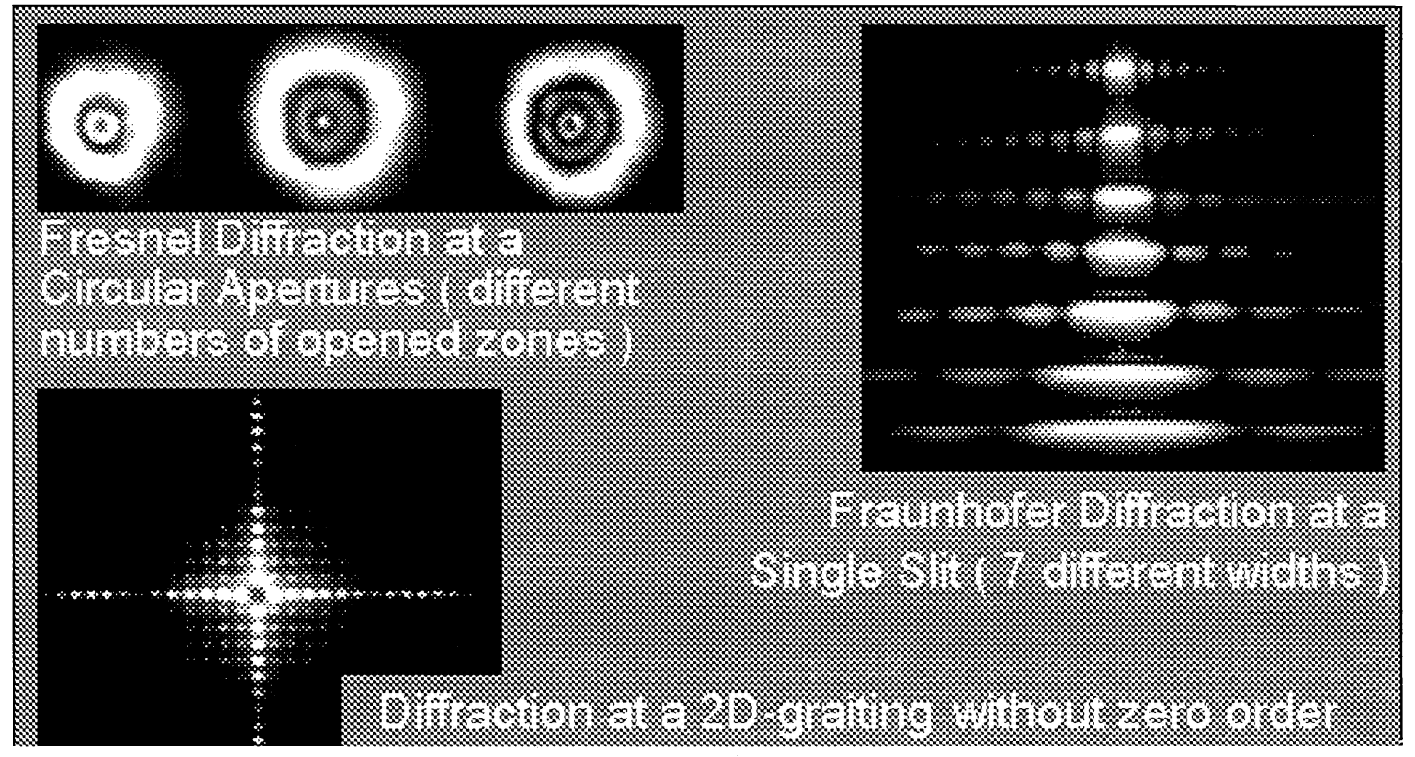

Fig. 5 Some real Images from Digital Files Collection: Diffraction patterns 
existing test- and check-files for current control tests to final tests. We put forward a preposition that total time limit should be from 60 minutes for final term testing to 120 minutes for final physics course testing for third year students. Personal results, collected by CheckShell can be taken into consideration on final examinations, but of course, can not replace them. Although an average score for the certain student for many tests and examination mark is usually in strong connection.

\section{GUIDE-PROGRAMS}

The first type of guide-programs is the real laboratory practice programs - LabShell (for students, see Fig.3) and LabMaker (for teachers). The software is fulfilled so that the laboratory work would be executed in three regimes. The first one is a manual doing without PC support. The second regime is carried out in the case when PC plays the role of an instructor and notebook. At last the rest regimes have got an automated input of dates from digital devices and remote control of some optical units - stepmotors modules from OMEL laboratory [2].

Simultaneously with the labs work doing students can open the other type of guide-programs - GuideShell (see Fig.4, the example of Ray Optics exercise) with theory information and short methods explanation All GuideShells include the pictures of optical circuits or real images from Digital Files Collection (see Fig.5). In some of labs students have to use Microsoft software for image processing and investigate the properties of specific images: shape and contrast of interference stripes, diffraction distributions, photoelastic phenomena etc.

The simulating program is destined to show a qualitative and numerical changing of dependencies on the PC screen. They may be studied on large varying of parameters for physical phenomena that haven't an apparatus realization. Each from these programs is finished up completely and may be used independently. Some simulating programs can represent the laboratory exercises in case when there's rendered on the screen of display an optical scheme that has the connection of digital devices with the PC. Files of dates to be input beforehand and to be treated enable to give an impressive illusion of laboratory work doing on the monitor. These regimes and programs became necessary for the students to do a laboratory work was missed during the fixed studies for some reasons.

The great number of our students take an active part in creation, testing and using new optical software (two last authors of this report are students of 5 and 3 year studying). They successfully apply their knowledge in computer science and optics not only for education purposes, but also for carry out the valuable investigations in modular optical laboratory: fractal scattering, computer generated holograms, nondestructive inspections, IR-vision, optical methods in ecology etc. Each students generation creates own original software and the quantity of our optical programs, pictures and image files is increasing constantly.

\section{REFERENCES}

1. S.C. Stafeev, Y.L. Kolesnikov, A.V. Smirnov, Concept of Modern Optical Training Labs for Optical Engineering Students, same volume.

2. S.C.Stafeev, Y.L.Kolesnikov and others, Training computerized laboratory for wave and quantum optics course, Proc. of the Conference "Education \& Training in Optics", p.192-196, Hungary, Pecs, 1993. 